\title{
Fabrication and Actuation of IPMC based on PVA/ SSA /5-SSIPA
}

\author{
Yunheng Yao \\ Department of Chemical \\ Engineering, Yanbian University \\ Jilin, 133002, China \\ hengheng3456@126.com
}

\author{
Yuexian Liu* \\ Department of Chemical \\ Engineering, Yanbian University \\ Jilin, 133002, China \\ *Corresponding author: \\ 1xliu@ybu.edu.cn
}

\author{
Huiqin Lian \\ Department of Chemical \\ Engineering, Yanbian University \\ Jilin, 133002, China \\ hqlian@ybu.edu.cn
}

\begin{abstract}
A novel ionic polymer based on crosslinked poly(vinyl alcohol) - succinic acid -5-Sulfoisophthalic acid sodium salt (PVASS) was prepared using solution casting method and the electromechanical performance was studied for the first time. The PVASS membranes were characterized using Fourier transform infrared(FT-IR) and their physical properties in terms of linear expansion, water uptake and ion-exchange capacity were tested. The results showed that the composition of PVASS membranes influenced their physical properties as well as the electromechanical performance.
\end{abstract}

Keywords-crosslinked; Ionic polymer-metal composites; actuation

\section{INTRODUCTION}

Ionic polymer is a kind of polymer electrolyte. It can be used in the field of lithium-ion batteries, fuel cell, biomaterials and electroactive polymer (EAP) actuator ${ }^{[1]}$. Among EAP actuators, ionic polymer metal composites (IPMCs) have the character of large bending deformation under low voltage of electricity ${ }^{[2]}$, their application involved in dynamic sensors, robotic actuators and artificial muscles ${ }^{[3-5]}$.

IPMCs consist of a thin ionic polymer membrane with metal electrodes plated chemically on both faces. IPMCs bend to the anode side when they subject to a voltage due to the migration of cation in the polymer. Therefore, Ionic polymer plays an important role in IPMCs. Lots of scientists are interested in study of syntheses and structure of ionic polymer. Among them, Nafion has a strong mechanical strength, high proton conductivity, and chemical stability, it has been widely used in preparation IPMCs ${ }^{[6-7]}$. However, the Nafion is fluorinated polymer, its high price and less environmentfriendly, limit its applications ${ }^{[8]}$. Therefore, hydrocarbon ionic polymers such as sulfonated PVDF/PVP/PSSA ionic membranes ${ }^{[9]}$, sulfonated polyetherimide (SPEI) [10-11], sulfonated polystyrene(sPs) $)^{[12-14]}$, sulfonated poly(vinyl alcohol) membrane ${ }^{[15-18]}$ poly((t-butyl-styrene)-b-(ethylene-rpropylene)-b-(styrene-r-styrene sulfonate)-b-(ethylene-rpropylene)-b-(t-butyl-styrene)) ionic membrane (tBS-EP-SSEP-tBS; SSPB) ${ }^{[19]}$ have been synthesized .

In this study, a novel ionic polymer, PVA/ SSA /5-SSIPA, was synthesized using one-step method through esterification. Polypyrrole was used to make electrodes ${ }^{[20-21]}$. The

This work was supported by the Natural Science Foundation of China (NSFC, No. 51063009), the Scientific Research Foundation for the Returned Overseas Chinese Scholars and State Education Ministry. composition of membranes influences the properties of membranes. The relative properties in term of mechanism and actuation are under study.

\section{EXPERIMENTAL SECTION}

\section{A. Material}

Polyvinyl alcohol $1750 \pm 50$ (PVA) was purchased from Sinopharm Chemical Reagent Co., Ltd, China. SSA (70 \% in water) and Sodium p-toluenesulfonate analytical grade AR , were provided by Tianjin Heowns Biochemical Technology Co., Ltd, China ; 5-Sulfoisophthalic acid sodium salt (5SSIPA) and Pyrrole were purchased from Aladdin reagent Shanghai crystal Pure reagent Co., Ltd. China; $\mathrm{FeCl}_{3} \cdot 6 \mathrm{H}_{2} \mathrm{O}$, AR, was purchased from Tianjin Guangfu Technology Development Co., Ltd, China ;

B. Preparation of partially crosslinked s-PVA membranes and 5-SSIPA

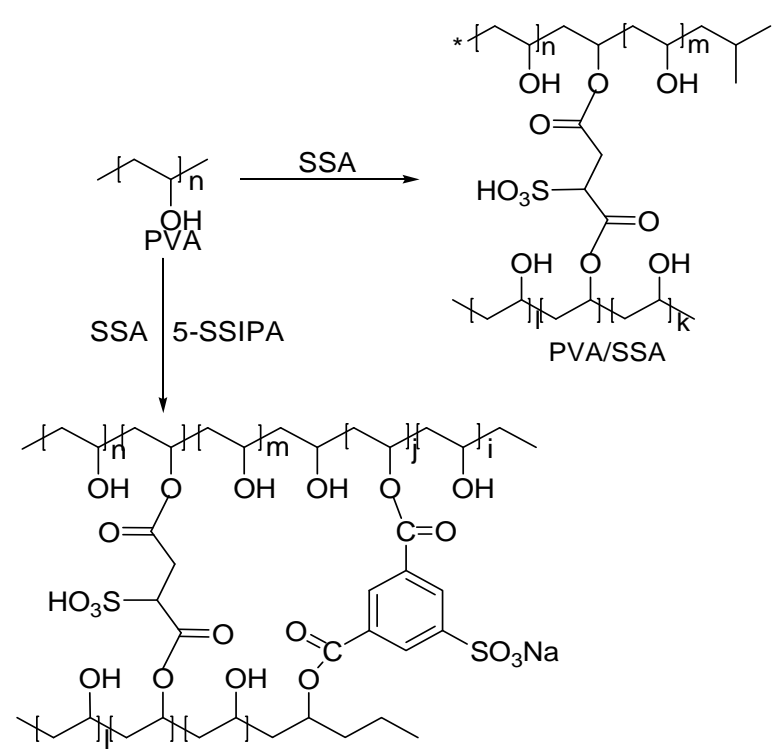

Fig.1 The synthetic scheme of PVAS and PVASS

The base membrane of crosslinked PVAS membrane was obtained using solution casting method through esterification 
of PVA and SSA. The details of the procedure were as follows: PVA solution of $10 \mathrm{wt} . \%$ was obtained by dissolving PVA in deionized water and stirring at $90{ }^{\circ} \mathrm{C}$ for $3 \mathrm{~h}$. The resulting PVA solution was mixed with SSA in mass ratio of $2: 1$.under stirring at room temperature for $24 \mathrm{~h}$. Following, the homogeneous solution was poured into a glass mold, heated at $40{ }^{\circ} \mathrm{C} 48 \mathrm{~h}$ and annealed at $80^{\circ} \mathrm{C}$ for $1.5 \mathrm{~h}$ to induce crosslinkages between PVA chains. The obtained membrane (PVAS) were activated and purified by $1 \mathrm{M} \mathrm{HCl}$ solution. And unreacted additives in the membranes were removed. Using the same procedure, membranes of PVASS6, PVASS17, PVASS 21 and PVASS25 were made with adding 5-SSIPA in weight percentage of $6 \%, 17 \%, 21 \%, 25 \%$ respectively. Fig. 1 shows the synthetic scheme for the PVAS and PVASS.

\section{Preparation of electrodes}

Polypyrrole were synthesized on the both sides of membranes as organic electrodes. A calculated amount of $\mathrm{FeCl}_{3} \cdot 6 \mathrm{H}_{2} \mathrm{O}$ and Sodium p-toluenesulfonate were dissolved in $100 \mathrm{ml}$ deionized water. Then the membranes were put into the solution, meanwhile $1 \mathrm{ml}$ of pyrrole was dropped into the solution under stirring for $0.5 \mathrm{~h}$ at $0^{\circ} \mathrm{C}$. Then the membranes were washed twice using distilled water.

\section{CHARACTRACTERIZATION}

Fourier transform infrared spectrometer (FT-IR Nicolet 6700) was used to characterize the chemical structures of membranes in this study. The Linear expansion (LE) and Water uptake (WU) property were determined by measuring the change in size and weight before and after water sorption. The Linear expansion (LE) and Water uptake (WU) were obtained using Eq.(1) and (2) respectively.

$$
\mathrm{WU}=\frac{W w e t-W d r y}{W d r y} \times 100(\%)
$$

Where $\mathrm{W}_{\text {wet }}$ and $\mathrm{W}_{\text {dry }}$ denote weight of the wet and dry membranes, respectively.

$$
\mathrm{LE}=\frac{L w-L d}{L d} \times 100(\%)
$$

Where $\mathrm{L}_{\mathrm{w}}$ and $\mathrm{L}_{\mathrm{d}}$ denote the length of the wet and dried membranes, respectively.

The Ion exchange capacity (IEC) was measured using titration method. A certain amount of membrane was immersed in $50 \mathrm{ml}$ of $1 \mathrm{M} \mathrm{HCl}$ solution in order to convert the sulfonate group to $\mathrm{H}^{+}$form. The membranes were then washed with deionized water to remove excess $\mathrm{HCl}$ and equilibrated with $50 \mathrm{ml}$ of $1 \mathrm{M} \mathrm{NaCl}$ solution at room temperature for 24h.The IEC(meq $\left./ \mathrm{g}^{-1}\right)$ of the membranes was determined by titration with $0.01 \mathrm{M} \mathrm{NaOH}$ solution using phenolphthalein as an indicator. The IEC of the membranes was calculated using Eq.(3)

$$
\mathrm{IEC}=\frac{0.01\left(\mathrm{~V}_{\mathrm{O}, \mathrm{NaOH}}-V_{E, \mathrm{NaOH}}\right)}{W_{d r y}}
$$

Where $\mathrm{V}_{\mathrm{O} . \mathrm{NaOH}}$ is the volume of $\mathrm{NaOH}$ at the beginning of titration, $\mathrm{V}_{\mathrm{E}, \mathrm{NaOH}}$ is the volume of $\mathrm{NaOH}$ after equilibrium, and $\mathrm{W}_{\text {dry }}$ is the weight of dry membrane $(\mathrm{g})$.

Electromechanical property was carried out by using a Labview-based system device. The IPMC was stimulated by programmable power. The programmable power was composed with an MCU of Mega8, digitally controlled potentiometer, adjustable voltage regulator AS1117 and relay, and provided an electrical signal with periods of $1 \mathrm{~s}$ to $5 \mathrm{~min}$, voltage of \pm 1.25 to $\pm 5 \mathrm{~V}$, and square wave. For the actuation measurement, specimen samples with dimensions of $30 \mathrm{~mm}$ length, $5 \mathrm{~mm}$ width and $0.25-0.35 \mathrm{~mm}$ thickness were prepared including the $10 \mathrm{~mm}$ of the electrode contact area. The measurements were carried out in air at room temperature after hydration in the $0.1 \mathrm{M} \mathrm{LiCl}$ water for $24 \mathrm{~h}$.

\section{RESULTS AND DisCUSSIONS}

The FTIR spectra of PVAS and PVASS were shown in Figure 2. The FT-IR spectrum of PVAS showed a broad band at $3340 \mathrm{~cm}^{-1}$ corresponding to the stretching vibration of $-\mathrm{OH}$. The bands at 1740 and $1080 \mathrm{~cm}^{-1}$ were associated with stretching of the $\mathrm{C}=\mathrm{O}$ and $\mathrm{C}-\mathrm{O}$ stretching vibrations respectively.

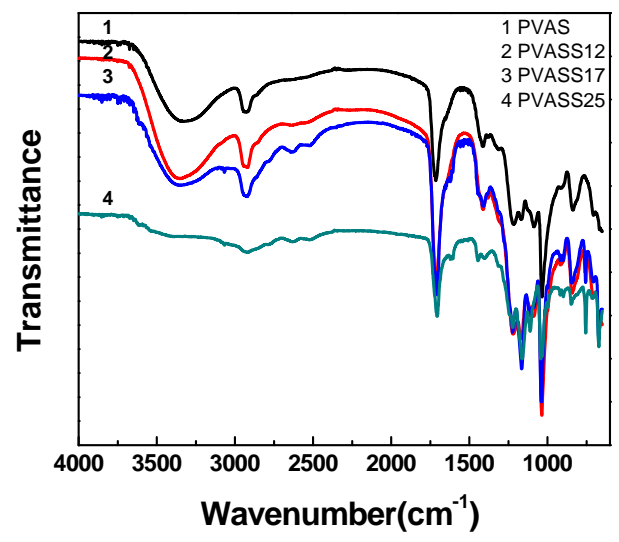

Fig. 2. FT-IR spectra of PVAS and PVASS.

From the spectra of PVASS membranes, it was noticed that the intensity of $-\mathrm{OH}$ decreased with the addition of SSA and 5-SSIPA indicating the reaction between PVA, SSA and 5-SSIPA. At the same time, they showed a quite different. character by the presence of new bands. The brands at 910 and $760 \mathrm{~cm}^{-1}$ were associated with bending vibration of benzene. The FTIR spectra of PVASS confirmed that 5SSIPA was introduced to the membranes successfully.

The physical properties of PVAS and PVASS membranes in terms of length expansion, water uptake and ionic exchange capacity as function of 5-SSIPA content were provided in Table 1. It showed that the values of LE and water uptake increase with 5-SSIPA content increase. When the content of 5-SSIPA was $17 \mathrm{wt} \%$, the membrane exhibited the highest value of length expansion of $224 \%$, water uptake of $353 \%$. The corresponding values for the PAVS membrane were 
$125 \%$ and $144 \%$ and 2.52 , respectively. However, when 5SSIPA concentration in the membranes was higher than $17 \%$, the values of LE and water uptake decreased to $178 \%, 302 \%$ of PVASS 21 and $189 \%, 247 \%$ of PVASS 25 . As for the value of IEC, the highest one was PVASS25 of $3.16 \mathrm{meq} / \mathrm{g}$, much higher than that of PVAS of $2.52 \mathrm{meq} / \mathrm{g}$. Therefore, the content of 5-SSIPA influenced the crosslink degree of membrane. The structure and mechanism are under studying.

TABLE 1 PHYSICAL PROPERTIES OF PARTIALLY CROSSLINKED S-PVA MEMBRANES

\begin{tabular}{|c|c|c|c|c|c|c|}
\hline & PVAS & PVASS6 & PVASS12 & PVASS17 & PVASS21 & PVASS25 \\
\hline LE(\%) & 125 & 145.4 & 156 & 224 & 178 & 189 \\
\hline WUP(\%) & 144 & 210 & 250 & 353 & 302 & 247 \\
\hline IEC(meq/g) & 2.52 & 2.61 & 2.17 & 1.89 & 2.57 & 3.16 \\
\hline
\end{tabular}
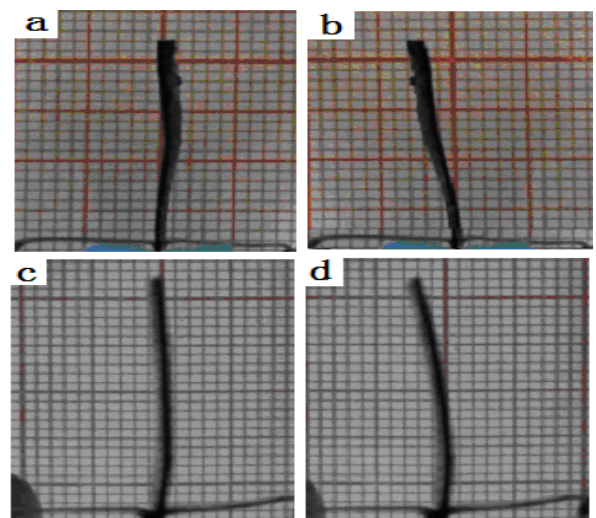

Fig . 3 . Displacement of an IPMC based on PVAS and PVASS membranes under electrical field: PVAS under (a) 0V and (b) 3V ; PVASS21 under (c) $0 \mathrm{~V}$ and (d) $3 \mathrm{~V}$.

The actuation of the PVAS and PVASS membranes was measured using a cantilevered actuation setup. The deflection of the membrane was evaluated by fixing one end of the membrane in the apparatus and measuring the movement of the free end, as shown in Fig.3. For comparison, PVAS and PVASS21 were selected for actuation test. Fig. 3a showed that the PVAS based IPMC actuators at $0 \mathrm{~V}$ had no deflection. When a voltage of $3 \mathrm{~V}$ was used to drive the IPMC actuator, it exhibited a tip displacement of $2 \mathrm{~mm}$ (Fig. 3b). From Fig. 3c and $3 \mathrm{~d}$, the IPMC based on PVASS21 showed the similar electromechanical performance as that of PVAS.

\section{$\mathrm{V}$ CONCLUSIONS}

Novel ionic polymer PVAS and PVASS were synthesized successfully. The physical properties of ion-exchange capacity, water uptake and linear expansion of the membrane were tested. IPMCs based on PVAS and PVASS were fabricated and their actuation behavior was tested. The result showed that the physical properties of membrane depended on the composition of the membranes. The IPMC based on PVAS and PVASS showed similar actuation performance.

\section{REFERENCES}

[1] Mohsen Shahinpoor and Kwang J Kim, Ionic polymer-metal composites: I. Fundamentals, Smart Mater. Struct. 10 (2001) 819-833.
[2] Kwang J Kiml and Mohsen Shahinpoor, Ionic polymer-metal composites: II. Manufacturing techniques, Smart Mater. Struct. 12 (2003) 65-79.

[3] Mohsen Shahinpoor and Kwang J Kim, Ionic polymer-metal composites: III. Modeling and simulation as biomimetic sensors, actuators, transducers, and artificial muscles, Smart Mater. Struct. 13 (2004) 1362-1388.

[4] Mohsen Shahinpoor, and Kwang J Kim, Ionic polymer-metal composites: IV. Industrial and medical applications, Smart Mater. Struct. 14 (2005) 197-214.

[5] Elaine Biddiss, Tom Chau, Electroactive polymeric sensors in hand prostheses: Bending response of an ionic polymer metal composite, Medical Engineering \& Physics 28 (2006) 568-578.

[6] Ren-Jei Chung, Tsung-Shune Chin, Li-Chun Chen, Ming-Fa Hsieh, Preparation of gradually componential metal electrode on solutioncasted Nafion membrane, Biomolecular Engineering 24 (2007) 434-437.

[7] Kumar Krishen, Space applications for ionic polymer-metal composite sensors, actuators, and artificial muscles, Acta Astronautica 64 (2009) 1160-1166.

[8] S. Holmberg, P. Holmlund, R. Nicolas, C.E. Wilen, T. Kallio, G. Sundholm, F. Sund-holm, Versatile synthetic route to tailor-made proton exchange membranes for fuel cell applications by combination of radiation chemistry of polymers with nitroxide-mediated living free radical graft polymerization, Macromolecules 37 (2004) 9909-9915.

[9] Varij Panwar, Kyoungrae Cha, Jong-Oh Park, Sukho Park, High actuation response of PVDF/PVP/PSSA based ionic polymer metal composites actuator, Sensors and Actuators B 161 (2012) 460-470.

[10] M. Rajagopalan, J.H. Jeon and I.K. Oh: Sens. Actuators B Vol. 151 (2010), p. 198.

[11] M. Rajagopalan and I.K. Oh: ACS Nano. Vol. 5 (2011), p. 2248.

[12] Mohammad Luqman, Jang-Woo Lee, Kwang-Kil Moon, Young-Tai Yoo, Sulfonated polystyrene-based ionic polymer-metal composite (IPMC) actuator.Journal of Industrial and Engineering Chemistry 17 (2011) 49-55

[13] Zheng Chen, Tae I. Um, Hilary Bart-Smith A novel fabrication of ionic polymer-metal composite membrane actuator capable of 3-dimensional kinematic motions. Sensors and Actuators A 168 (2011) 131-139.

[14] Yongmoon, Kwon,Judit E.Puskas ,Investigation of the effect of reaction conditions on the synthesis of multiarm-star polyisobutylene-polystyene block copolymers, European Polymer Journal 40(2004)119-127.

[15] K.D.McKeon-Fischer, D.H. Flagg, J.W. Freeman, Poly(acrylic acid)/poly(vinyl alcohol) compositions coaxially electrospun with poly(3-caprolactone) and multi-walled carbon nanotubes to create nanoactuating scaffolds, Polymer 52 (2011) 4736-4743.

[16] Chi-An Dai, Chun-Jie Chang, An-Cheng Kao, Wei-Bor Tsai, WenShiang Chen, Wei-Ming Liu, Wen-Pin Shih, Chien-Ching Ma, Polymer actuator based on PVA/PAMPS ionic membrane: Optimization of ionic transport properties, Sensors and Actuators A 155 (2009) 152-162.

[17] Jang-Woo Lee, Ji-Hye Kim, Nam Seo Goo, Jae Yeol Lee, Young-Tai Yoo1, Ion-Conductive Poly(vinyl alcohol)-Based IPMCs, Journal of Bionic Engineering 7 (2010) 19-28.

[18] Alan K. Phillips, Robert B. Moore, Ionic actuators based on novel sulfonated ethylene vinyl alcohol copolymer membranes, Polymer 46 (2005) 7788-7802.

[19] Jang-Woo Lee, Soon Man Hong, Joohoon Kim, Chong Min Koo, Novel sulfonated styrenic pentablock copolymer/silicate nanocomposite membranes with controlled ion channels and their IPMC transducers. Sensors and Actuators B:2012,xxx-xxx.

[20] Rauno Temmer, Indrek Must, Friedrich Kaasik, Alvo Aabloo, Tarmo Tamm Combined chemical and electrochemical synthesis methods for metal-free polypyrrole actuators, Sensors and Actuators B 166-167 (2012) 411-418.

[21] Kenneth K.C. Lee , Nigel R. Munce , Tina Shoa , Luc G. Charron , Graham A.Wright, John D. Madden, Victor X.D. Yang, Fabrication and characterization of laser-micromachined polypyrrole-based artificial muscle actuated catheters, Sensors and Actuators A 153 (2009) 230236. 